\title{
Energy dissipation mechanism of a non-obstructive particle damper based on DEM simulation
}

\author{
Fan $\mathrm{Su}^{1}$, Hang Zhang ${ }^{1}$ and Zhongjun Yin ${ }^{1, a}$ \\ ${ }^{1}$ School of mechanical engineering, University of science and technology Beijing, Beijing 100083, China
}

\begin{abstract}
The Non-obstructive Particle Damping (NOPD) has been widely used as an effective passive damping technique. However, the understanding of energy dissipation mechanism and meso-scale behaviors of granular system in NOPD is not in-depth enough. In this paper, the damping effectiveness of NOPD was analyzed combining with the motion modes of granular system and the energy dissipation mechanism was illustrated at the meso-scale. The results shows that the different motion states and meso-structure leading to the variations of the damping effectiveness. Generally, the NOPD has the optimal damping effectiveness in the buoyancy convection state. The purpose of this paper is to provide better understanding and theory support for the design and application of NOPD.
\end{abstract}

\section{Introduction}

The Non-obstructive Particle Damping (NOPD) has been widely used as an effective passive damping technique in aerospace [1, 2], mechanical engineering [3-5] and construction[6-8] fields for its simplicity and high efficiency. The mechanism of NOPDs for reducing the vibrations is based on the dissipative nature of the combined effects of inelastic collisions and frictional losses when the particles in the cavity collide and rub with themselves and the cavity walls $[9,10]$.

Recent studies on NOPD mainly focused on the vibration damping effectiveness and the contributions to the modal damping of structures based on experimental investigation and numerical simulation $[5,9,11]$. However, the understanding of energy dissipation mechanism and meso-scale behaviors of granular system in NOPD is not in-depth enough. In fact, granular matter has various and special rheology behaviors, just as the solid state, liquid state and gas state [12]. Only a few researches concerned the characteristic of energy dissipation. These impede the further development and application of NOPD.

Discrete element method (DEM) has gradually become an important tool in the research of granular system with the continuous improvement of DEM and development of computer technology $[13,14]$, which can track the stresses and motions of individual particles in time, providing sufficient information for analyzing the principles of energy dissipation in the granular system of NOPD.

In this paper, DEM was applied in the simulation of NOPD. The damping effectiveness of NOPD was analyzed combining with the motion modes of granular system to obtain the influence of different vibration conditions on damping property and demonstrate the energy dissipation mechanism of

\footnotetext{
${ }^{a}$ Corresponding author : yinzhongjun@ustb.edu.cn
} 
granular system at the meso-scale. The purpose of this paper is to provide better understanding and theory support for the design and application of NOPD.

\section{Simulation model and parameters}

In this work, a 3D scale granular system of NOPD model (Fig. 1) was built. It consisted of a closed Perspex cylindrical container $\left(\mathrm{D} \times \mathrm{H}=26 \times 78 \mathrm{~mm}^{2}\right)$ filled with $500(\mathrm{~d}=3.5 \mathrm{~mm})$ steel spheres. The material properties used in the simulation are based on the references [12], which is listed in Table 1.

The container was subjected to vertical sinusoidal excitation. The two control parameters were excitation frequency $f(\underset{\text { Tine: } 2 \mathrm{~s}}{15-90 \mathrm{~Hz})}$ and dimensionless excitation acceleration $\Gamma(1-30 \mathrm{~g})$.

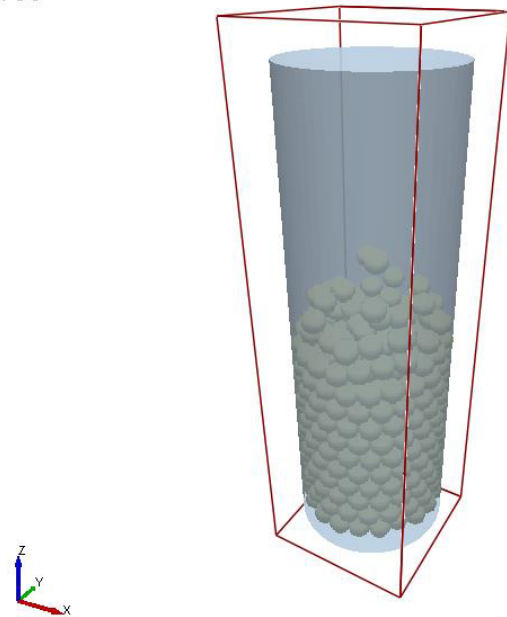

Figure 1. DEM model of the NOPD.

Table 1. Physical properties and parameters used in the DEM simulation.

\begin{tabular}{|c|c|c|c|}
\hline Parameter & Value & Parameter & Value \\
\hline Density of particle, $\rho_{p}\left(\mathrm{~kg} / \mathrm{m}^{3}\right)$ & 7800 & Density of container, $\rho_{c}\left(\mathrm{~kg} / \mathrm{m}^{3}\right)$ & 1190 \\
\hline Elasticity modulus of particle, $E_{p}(\mathrm{~Pa})$ & $206 \mathrm{e}+09$ & Elasticity modulus of container, $E_{c}(\mathrm{~Pa})$ & $3.3 \mathrm{e}+09$ \\
\hline Poisson ratio of particle, $v_{p}$ & 0.3 & Poisson ratio of container, $v_{\mathrm{c}}$ & 0.37 \\
\hline Diameter of particle, $d(\mathrm{~m})$ & 0.0035 & Coefficient of restitution, $e$ & 0.92 \\
\hline Total quantity of particles & 500 & Coefficient of static friction, $\mu_{\mathrm{s}}$ & 0.3 \\
\hline Time step, $\Delta t(s)$ & $2.07 \mathrm{e}-07$ & Coefficient of rolling friction, $\mu_{\mathrm{r}}$ & 0.01 \\
\hline Total time of each simulation, $t(s)$ & 2 & & \\
\hline
\end{tabular}

\section{Results and Discussion}

\subsection{Phase diagrams}

When containers filled with granular material are subjected to vertical vibrational motion, a series of rheology behaviors were observed. Based on the analysis of the simulation results, the motion modes 
of granular system were divided into seven different ones including solid-like state, local fluidization, global fluidization, convection [15], Leidenfrost effect [16], buoyancy convection and bouncing bed, which are shown in Fig. 2.

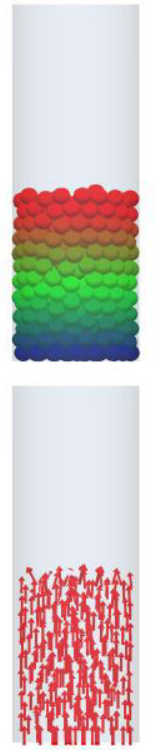

a)
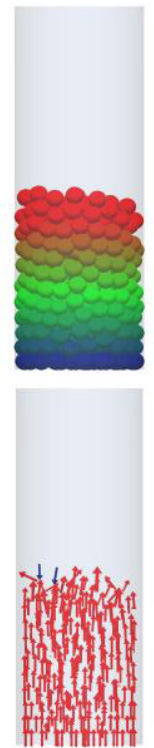

b)
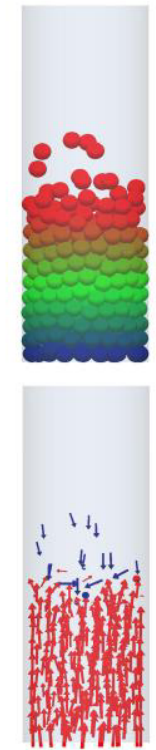

c)
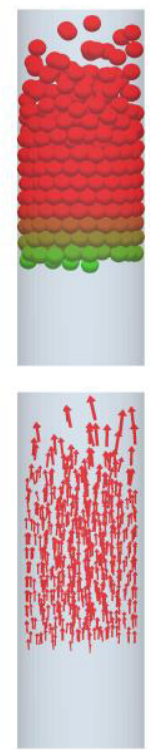

d)
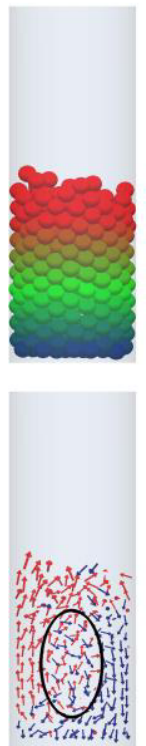

e)
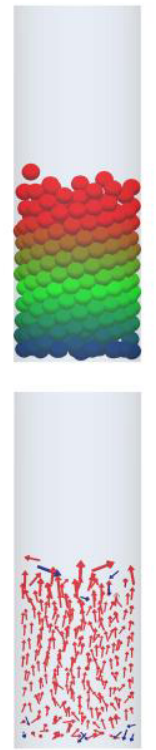

f)
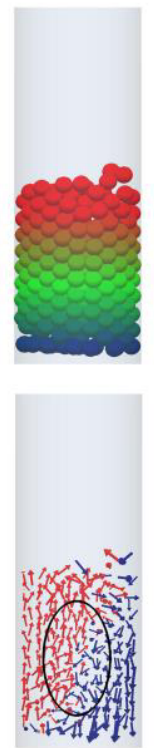

g)

Figure 2. Snapshots of particle motion modes and the corresponding velocity vector: a) solid-like state, b) local fluidization, c) global fluidization, d) bouncing bed, e) convection, f) Leidenfrost effect, g) buoyancy convection

When the excitation acceleration is below 1.2g, damping particles are in solid-like state (Fig.2 a)), there is almost no relative motion between particles and between particles and the wall, and the whole granular system looks like an added static mass.

When the excitation acceleration reached $1.2 \mathrm{~g}$, the phase transition of granular materials from solid to liquid happened. Particles which are closer from the top surface of the granular bed become fluidized while particles in the lower granular layer are still relatively motionless. Fig. $2 \mathrm{~b}$ ) shows the slight relative motions between particles in the top granular layers.

With the increasing of amplitude or frequency, the fluidized granular layers were expanding to the whole granular system, and the relative motions between particles occurred in all granular layers. The particles are loose and reached global fluidization state (Fig. 2 c)).

Under the condition of large amplitudes, particles in the granular system behave as inelastic bouncing balls impacting chaotically and show series of phenomena of subharmonic bifurcation [17]. It should be noted that the bouncing bed state in this paper is defined as the condition for which the top granular layer completely contacts with the top of container (Fig. $2 \mathrm{~d}$ )).

When convection occurs, there is not only relative motion between particles but also the exchange of the particle position between granular layers. As can be seen from Fig. 2 e), the velocity vector distribution of granular system when $f=65 \mathrm{~Hz}$, there was a flowing convection rolls in granular layers.

Above a critical shaking strength, a cluster of particles is elevated and supported by a few fast particles underneath the cluster. This cluster exhibits an almost perfect hexagonal close packing, causing the density to be maximal at the floating cluster and distinctly lower above and below this cluster (Fig. 2 f)). The thickness of the dilute layer oscillates in time (never vanishing) due to the motion of the bottom, while the cluster floats steadily in the vicinities of their original positions [16]. This state appears on condition that both the frequency and vibration intensity are high.

The buoyancy convection (Fig. 2 g)) can be seen as a superpositional state of granular Leidenfrost effect and convection, i.e., convection occurs in suspended granular layers which are in Leidenfrost 
effect state [12]. Just like the convection, there is also a flowing convection rolls in suspended granular layers.

To directly observe the occurrence conditions of different motion modes in granular system, the phase diagram of damping particles in the NOPD under different excitation conditions was obtained, as depicted in Fig. 3.

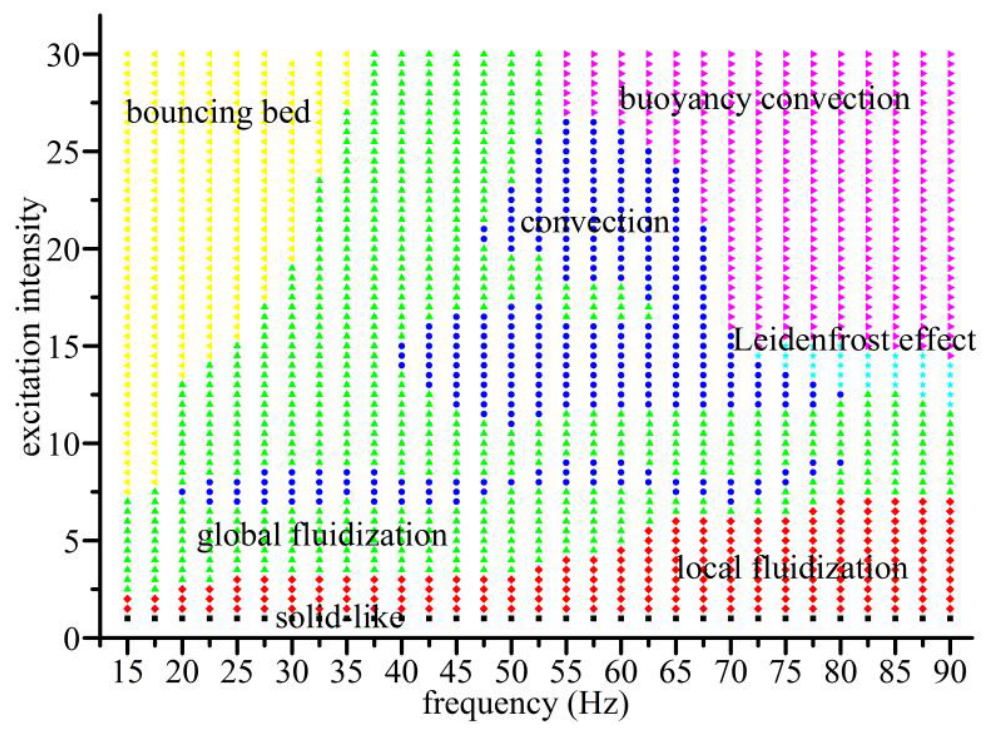

Figure 3. Phase diagrams of damping particles in NOPD

The transitions of different motion modes can be seen from the phase diagram obviously. It is notable that global fluidization state is the basis for transitions to other states and convection is a relatively unsteady state compared with other states, and the phase boundary of global fluidization state and convection is not as clear as others.

\subsection{Loss factor}

In this work, the damping efficiency is measured by the damping loss factor [18], which is given as

$$
\eta=\frac{E_{\text {dissipated }}}{E_{\text {maximum }}}
$$

where $E_{\text {dissipated }}$ is the dissipated energy in a cycle per radian, and $E_{\max \text { imum }}$ is the maximum energy stored in a cycle, which is approximately equal to the maximum kinetic energy in a cycle.

Four cases in Fig. 3 covering different processes of transition were selected, and the energy dissipation and the maximum kinetic energy along the excitation intensity for each case are plotted in Fig. 4 a) and b). As it can be seen, generally both the energy dissipation and kinetic energy increase with the increase of excitation intensity, and higher frequencies have a lower increase.

Fig. 5 shows the loss factor versus excitation intensity under different frequencies. Comparing the different curves, it can be observed that the particles under different frequencies offer best damping in different excitation condition. When the frequency is low, the loss factor presents the maximum value under the condition of low excitation intensity, the loss factor peaks around $3 \mathrm{~g}$ and then decreases shapely with the increase of the excitation intensity. After $20 \mathrm{~g}$, the loss factor gradually stabilizes at a constant value. When the frequency is high, the loss factor reaches another peak $(30 \mathrm{~g})$ and presents the maximum value under the condition of high excitation intensity. 

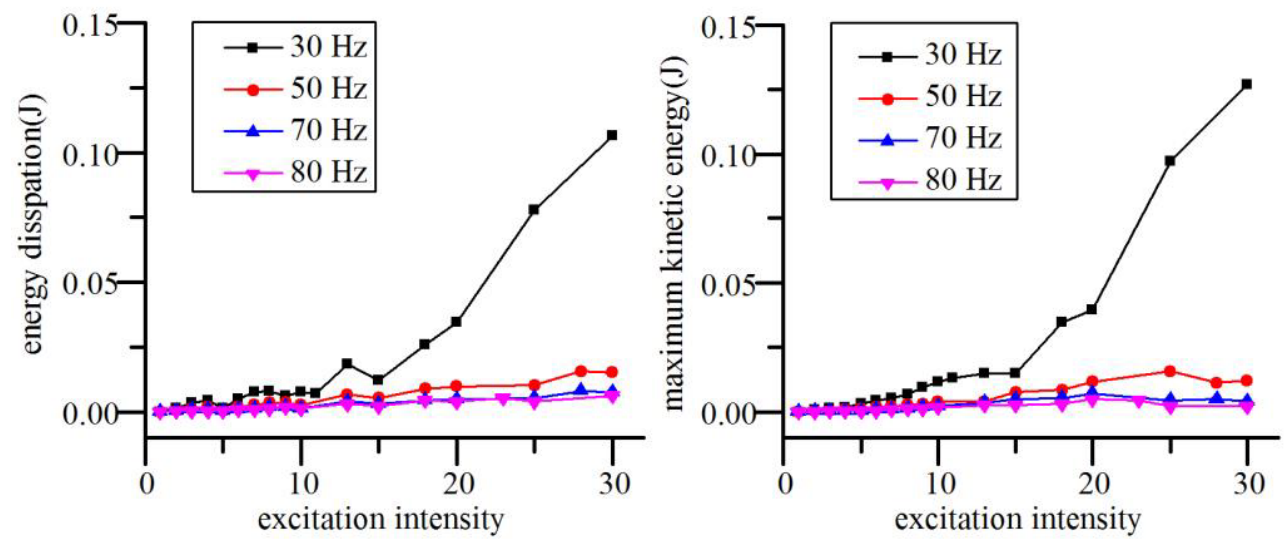

Figure 4. a) The energy dissipation in a cycle, b) the maximum kinetic energy in a cycle

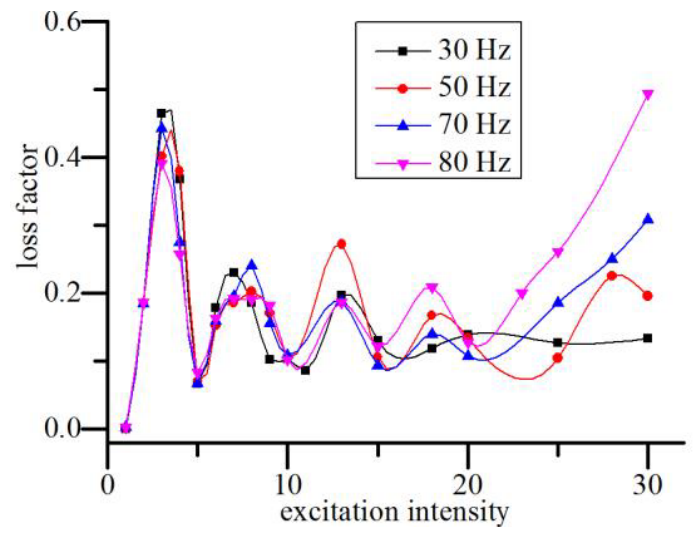

Figure 5. Loss factor versus excitation intensity under different frequency

Combined with the phase diagram, it can be concluded that the variations of loss factor are due to the motion modes transitions of the granular system.

When the solid-like state transits to local fluidization state, the higher the amplitude or frequency, the more violent the particles' motion, especially the particles which are closer from the top surface of the granular system, and the more active motion of particles in the local fluidization state results in more energy dissipation because the collisions between particles and the walls become more frequent. However, the maximum kinetic energy is still low in comparison with the energy dissipation, thus a sharp increase on the value of loss factor can be seen at about $3 \mathrm{~g}$.

The fluidization state will transit to bouncing bed state with the increase of the excitation intensity under low frequencies, where the increased opportunity of collisions and the severity of impacts dissipate a lot of energy. However, the loss factor would not increase infinitely along with the increase of excitation intensity. Most of the collisions happen between the particles and the walls of the container, and the impacts and motions of particles are too chaotic to reduce vibration because of the subharmonic bifurcation [17]. Therefore, the value of loss factor at $f=30 \mathrm{~Hz}$ is small and staying near 0.12 at large excitation intensity.

Compared to other states, convection is an unstable state, the phase boundary between it and the global fluidization state is not as obvious as other boundaries, and the convection shows period-like properties with excitation intensity and frequency, which occurs in a higher frequency with the increases of excitation intensity. The value of loss factor of convection has less difference with the global fluidization state, but the periodic switch of directivity of convective motion in granular system causes more collision chances, which means that in convection state, the loss factor of NOPD is slightly larger than the value in the global fluidization state. 
The Leidenfrost effect and the buoyancy convection state appear under the condition of high frequency and large vibration intensity, and a cluster of particles is elevated and supported by a few fast particles underneath the cluster. There will be an extra energy dissipation to stabilize the suspension of the granular layers except the collisions and frictions between particle-particle and particle-wall. Therefore, these two states have relatively large loss factor. But the meso-structure of suspended layer in the Leidenfrost effect and the buoyancy convection state is different. The suspended layer is in the solid-like state for the Leidenfrost effect that the meso-structure is an almost perfect hexagonal close packing, while is in the convection state for the buoyancy convection state. In analogy to the difference between the solid-like state and convection state, the energy disspation of collisions and frictions in the particle suspension layer in buoyancy convection is more than in the Leidenfrost effect state. Therefore, when NOPD plays the optimal damping effectiveness, particles in NOPD are in the buoyancy convection state.

\section{Conclusion}

In this work, the loss factor of particle damping was analyzed combing with the motion modes of granular system based on DEM, and the following conclusions are obtained:

1. The NOPD offers best damping in different vibration parameters. When frequency is low, the value of loss factor peaks at low excitation intensity. When frequency is high, the loss factor presents the maximum value at high excitation intensity.

2. The different motion states and meso-structure leading to the variations of the loss factor of granular system. Generally, the NOPD has the optimal damping effectiveness in the buoyancy convection state.

The purpose of this work is to provide a better understanding of the particle damping effectiveness and theory support for the design of the particle damper.

\section{References}

1. P. Lieber, D. Jensen, Trans. ASME, 67 (1945)

2. J.J. Moore, A. Palazzolo, R. Gadangi, T. Nale, S. Klusman, G. Brown, A. Kascak, J. Vib. Acoust., 117 (1995)

3. A. Paget, Engineering, 143 (1937)

4. E. Skipor, L. Bain, J. Mech. Des, 102 (1980)

5. W. Xiao, J. Li, S. Wang, X. Fang, J. Sound Vib., 366 (2016)

6. K. Ogawa, T. Ide, T. Saitou, J. Wind Eng. Ind. Aerodyn., 72 (1997)

7. J. Yao, J. Struct. Div., 98 (1972)

8. J.M. Kelly, R. Skinner, A. Heine, Bull. New Zealand Soc. Earthq. Eng., 5 (1972)

9. J.M. Bajkowski, B. Dyniewicz, C.I. Bajer, J. Sound Vib., 341 (2015)

10. H. Panossian, J. Vib. Acoust., 114 (1992)

11. B. Yao, Q. Chen, H. Xiang, X. Gao, Int. J. Mech. Sci., 80 (2014)

12. K. Zhang, T. Chen, X. Wang, J. Fang, J. Sound Vib., 364 (2016)

13. K. Mao, M.Y. Wang, Z. Xu, T. Chen, Powder Technol., 142 (2004)

14. M. Saeki, J. Sound Vib., 281 (2005)

15. E. Ehrichs, H. Jaeger, G.S. Karczmar, J.B. Knight, Science, 267 (1995)

16. P. Eshuis, K. van der Weele, D. van der Meer, D. Lohse, Phys. Rev. Lett., 95 (2005)

17. N. Mujica, F. Melo, Phys. Rev. E, 63 (2000)

18. M.B. Romdhane, N. Bouhaddi, M. Trigui, E. Foltête, M. Haddar, MSSP, 38 (2013) 